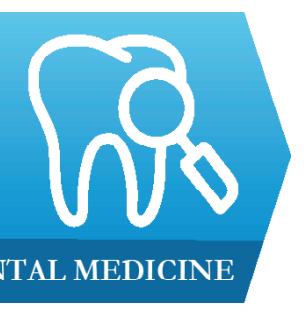

DENTAL MEDICINE

\section{Ligature induced periodontitis causes atherosclerosis in rat descending aorta: an experimental study}

Tudor Dimitriu ${ }^{1}, Z_{\text {sofia Daradics }}^{2}$, Șoimița Suciu ${ }^{3}$, Gabriel Armencea ${ }^{1}$, Cornel Cătoi ${ }^{2}$, Cristian Dinu ${ }^{1}$, Grigore Băciuț ${ }^{1}$, Sergiu Văcăraș ${ }^{1}$, Simion Bran ${ }^{4}$, Mihaela Băciuț ${ }^{1}$
1) Department of Oral and Maxillofacial Surgery, Iuliu Hatieganu University of Medicine and Pharmacy, Cluj-Napoca, Romania

2) Department of Pathology, University of Agricultural Sciences and Veterinary Medicine, Cluj-Napoca, Romania

3) Department of Physiology, Iuliu Hatieganu University of Medicine and Pharmacy, Cluj-Napoca, Romania

4) Department of Oral Rehabilitation, Iuliu Hatieganu University of Medicine and Pharmacy, Cluj-Napoca, Romania
DOI: $10.15386 / \mathrm{mpr}-1514$

Manuscript received: 27.06.2019

Received in revised form: 11.09.2019 Accepted: 28.09.2019

Address for correspondence: garmencea@gmail.com

\begin{abstract}
Introduction. In recent years a link between periodontitis and cardiovascular diseases has been proposed. Oxidative stress has been found to be involved in their pathophysiology.
\end{abstract}

Objectives. The aim of this study was to demonstrate that a wire ligature placed around the first mandibular molars of the rat causes inflammation in the periodontium and lipid deposits in the aorta, via oxidative stress.

Methods. Twenty male Wistar rats were divided randomly into two groups of 10: Control group and Periodontitis group. An orthodontic wire ligature was placed around the cervix of the first mandibular molar and was kept in place for 4 weeks. Clinical attachment loss was determined to assess the gingival recession. Aorta was harvested after 4 weeks and the malondialdehyde (MDA) levels in the aorta tissue homogenate were determined. Lipid deposits were evaluated using Oil Red staining and immunofluorescence under confocal microscopy. Total lipid deposits in the aorta were assessed using a computer morphometry system.

Results. Significant gingival recession was observed in the Periodontitis group in comparison with the Control group. Increased MDA levels were present in the aorta in the ligatured rats in comparison with the Control group. Significant lipid deposits were observed in the aorta of the Periodontitis group as opposed to the Control group.

Conclusions. The affixing of an orthodontic wire ligature produces gingival recession, increased oxidative stress and lipid deposits in the rat descending aorta.

Keywords: periodontitis, oxidative stress, atherosclerosis, ligature, rat

\section{Introduction}

Periodontitis is one of the most common inflammatory diseases of the oral cavity causing gingival recession and bone resorption that leads to tooth loss. Up to $90 \%$ of the world's population suffers from periodontal disease [1]

Atherosclerosis is one of the most important factors in the pathogenesis of cardiovascular diseases like myocardial infarction and stroke. It has a proven inflammatory component which triggers the activation of the immune response in the arteries [2].

Cardiovascular diseases and periodontitis share common risk factors such as smoking, age, diabetes mellitus, obesity and dyslipidemias [3]. This has led to the assumption that the two are linked by their inflammatory component and oxidative stress [4]. Systemic inflammation causes low density lipoproteins (LDL) in the endothelium and intima to oxidize and attract lymphocytes and macrophages, especially the proinflammatory phenotype M1 [5]. C reactive protein (CRP) is known to be involved in the atherosclerosis development. It has also been found in high levels in the serum and periodontal tissues in patients suffering from periodontitis. CRP has an important proinflammatory action and it increases the number of leukocytes. These release a high concentration of cytokines which are responsible for lesions 
in the endothelium, endothelial dysfunction and determine the migration of vascular smooth muscle cells in the intima of arteries. CRP opsonizes the LDL cholesterol facilitating the capturing of LDL particles by macrophage cells which transform into foam cells that are directly involved in the formation of atherosclerotic plaques [6,7].

Bacteria found in the periodontal pockets, such as Porphyromonas gingivalis have been shown to initiate or worsen atherosclerosis by activation of the innate immunity and by direct involvement of the mediators activated by the bacteria antigens [8]. The lipopolysaccharides (LPS) of the periodontopathogenic bacteria found in the serum further stimulates the monocytes and macrophages by binding to CD14, a receptor for LPS. This process allows the suppression of the immune response against the LPS [8-10]. The high concentration of LPS determines an acute inflammatory response which causes an alteration of the normal function of the osteoclasts and osteoblasts that leads to bone resorption [11].

Developing an experimental model of periodontitis that initiates atherosclerosis in the aorta of rats is of great importance in finding ways to better understand the pathophysiology and the involvement of oxidative stress in both diseases. In the literature there are studies describing the experimental model of periodontitis using silk or cotton ligatures around the maxillary molars or lower incisors of rats $[12,13]$. No studies have used an orthodontic wire around the mandibular molars.

The aim of this study was to demonstrate and elucidate the way that an orthodontic wire placed around the mandibular first molar induces periodontitis and atherosclerosis.

\section{Methods \\ Animals}

Twenty male Wistar Albino rats, weighing $400 \pm$ 50 grams, from the animal facility of the Iuliu Hatieganu University of Medicine and Pharmacy, Cluj-Napoca, Romania, were included in the study. The animals were kept in controlled conditions: temperature $22 \pm 2^{\circ} \mathrm{C}$, humidity 50 $\pm 10 \%$, light-dark cycle $12 \mathrm{~h}-12 \mathrm{~h}$ and had free access to tap water and solid food. The animals were weighed before and 4 weeks after the ligature was placed. No significant weight loss was recorded. Maintenance and all the procedures on the experimental animals were performed according to the guidelines for animal experiments set by the Ethical Committee of the Iuliu Hatieganu University of Medicine and Pharmacy, Cluj-Napoca, Romania, authorization number 377/16.10.2018. The animals were randomly divided into 2 groups of 10 animals per group: Control group and Periodontitis group.

\section{Induction of periodontitis}

The animals in the Periodontitis group were anesthetized using a mixture of Ketamine and Xylazine 2:1, injected intraperitoneally. A $0.4 \mathrm{~mm}$ diameter stainless steel orthodontic wire was passed through the space between the first and second mandibular molars and then tightened around the subgingival portion of the crown of the first molar. The wire was kept in place for 4 weeks.

\section{Tissue collection}

At the end of the 4 weeks the animals were sacrificed using an anesthesia overdose. Clinical attachment loss was assessed using a periodontal probe. There were two independent observers, double blind for the sake of reducing measuring inaccuracies. Assessment of the gingival recession was done in the mesiolingual aspect of the first mandibular molar after the removal of the ligatures by measuring the distance between the cement-enamel junction and the apical extent of the periodontal sulcus. The aorta was dissected from the lower thoracic region to the iliac bifurcation and was cleaned of adventitial fat.

\section{Aortic lipid deposits quantification}

The descending aorta was harvested and fixed in $20 \%$ paraformaldehyde and sent for histopathological and immunohistochemical examinations. Then it was opened longitudinally along the ventral side with fine scissors. The aorta was briefly rinsed in $70 \%$ ethanol, then staining with $0.5 \%$ Sudan IV and $35 \%$ ethanol- $50 \%$ acetone was performed for 6 minutes. Destaining was done with $80 \%$ ethanol for 5 minutes. Afterwards each aorta was placed on a flat surface and using an Olympus digital camera, digital images of the aortic surfaces were obtained. The images were analyzed using computer assisted morphometry software and the areas covered by lipids were expressed as percentages of the total surface area of the aorta.

\section{staining \\ Evaluation of aorta lipid deposits using Oil Red}

Frozen sections of aorta were prepared and mounted on slides. The slides were placed in propylene glycol solution for 2 minutes, then incubated in Oil Red O solution for 6 minutes. A mixture of $85 \%$ propylene glycol solution was prepared in distilled water and the tissue section was differentiated in the mixture for 1 minute. The slides were rinsed in 2 changes of distilled water and then incubated in Hematoxylin solution for 2 minutes. Tap water was then used to rinse the slides, followed by rinsing them with distilled water. The slides were then coverslipped using an aqueous mounting medium. The slides were examined using a standard microscope (Olympus) and under Texas Red filter (Texas Red Filter Cube Set - Olympus) using confocal microscopy (Zeiss LSM 710 confocal Laser Scanning unit using the standard ZEN software package.

\section{Malondialdehyde evaluation in the aorta}

The reagents provided in the kit (Lipid Peroxidation (MDA) Assay kit - Colorimetric/Fluorometric) were prepared according to the instructions and standard protocols. Aorta tissue homogenate was prepared and then washed in cold PBS. Lysis solution was prepared according to the standard protocols. The tissue was homogenized in $303 \mu \mathrm{L}$ Lysis Solution (Buffer + BHT) with a Dounce homogenizer sitting on ice, with $10-15$ passes. Then it was centrifuged at $13.000 \mathrm{x} \mathrm{g}$ for 10 minutes to remove insoluble material. 
The supernatant was collected. The prepared material was analyzed and absorbance was measured immediately with a fluorometric assay on a microplate reader at $\mathrm{Ex} / \mathrm{Em}=$ $532 / 553 \mathrm{~nm}$.

\section{Statistical analysis}

The data collected was entered into a Microsoft Excel data sheet and analyzed using MedCalc software for Windows, Version 18. Data was analyzed using the unpaired two-tailed Student's $t$-test to calculate the differences between the groups. The significance level was set if $P<0.05$.

\section{Results}

Increased gingival recession was observed after the removal of the ligature in the periodontitis group $(0.37$ $\mathrm{mm} \pm 0.2)$ as opposed to the control group $(0.09 \mathrm{~mm} \pm$ 0.07 ), $P<0.0006$ (Table I).

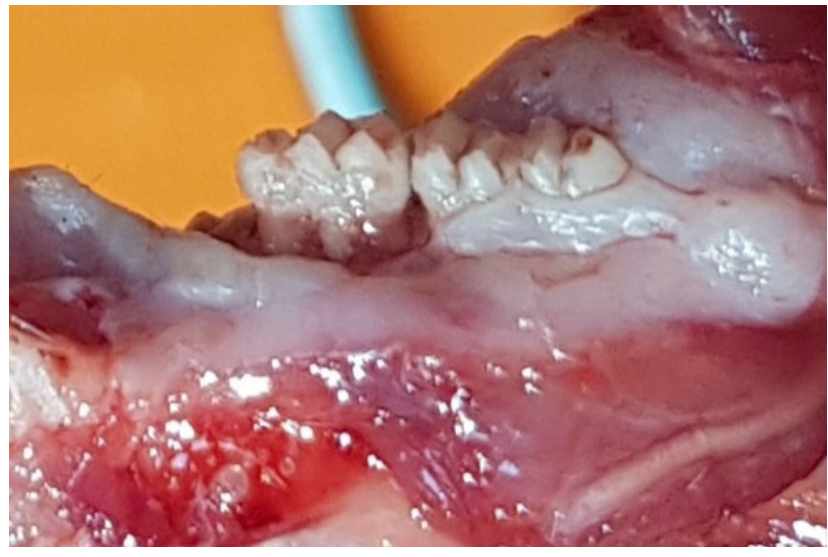

Periodontitis group

Figure 1. The gingival recession and bone loss associated with the ligature.

Table I. Average clinical attachment loss measured in millimeters.

Periodontitis group 0.37

In the Periodontitis group the epithelial insertion of the gingiva is situated in a more apical position where the ligature has been in contact with the first molar in comparison with the second molar. The exposure of the roots of the first molar indicates bone resorption associated with the wire ligature as opposed to the Control group in which no clinical signs of inflammation and gingival recession are identified (Figure 1).

After 4 weeks there was a significant increase in MDA levels in the descending aorta in the periodontitis group as compared to the control group $(P<0.002)$ (Figure 2$)$.

Oil Red dye staining shows the presence of lipid deposits in the descending aorta in the periodontitis group, in comparison to the lack of lipids in the control group (Figure 3).

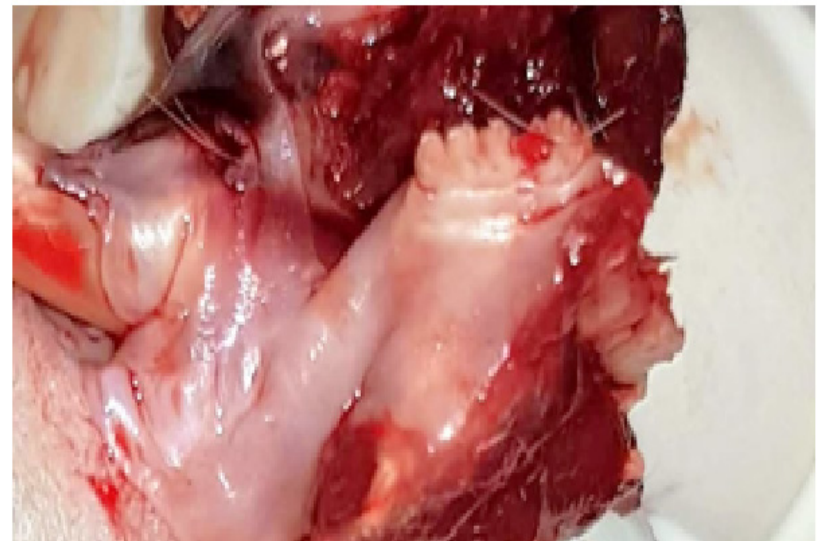

Control group

$4.41 \mathrm{nmol} / \mathrm{mg}$ prot

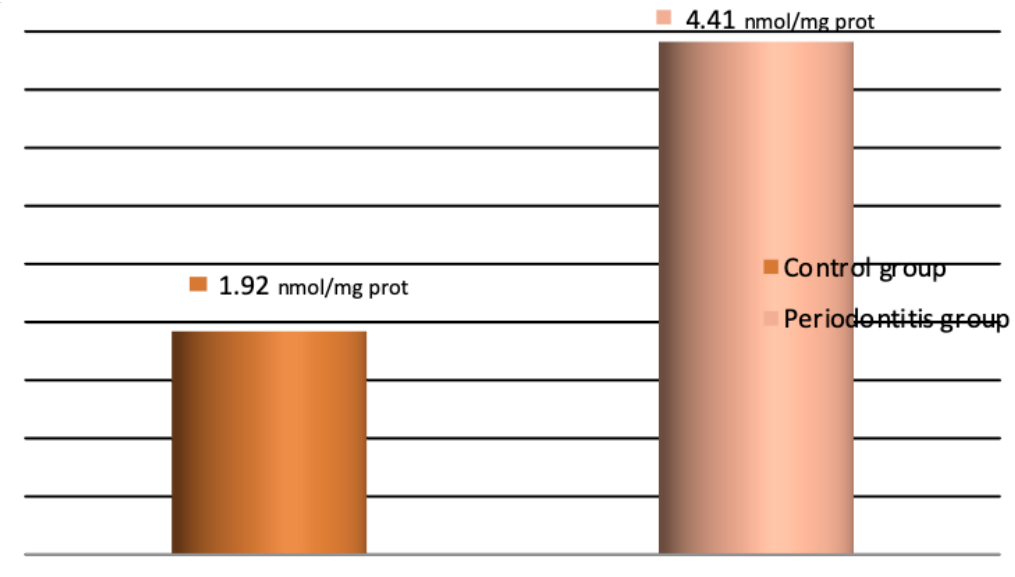

Figure 2. The levels of MDA in descending aorta homogenate at day 28. 


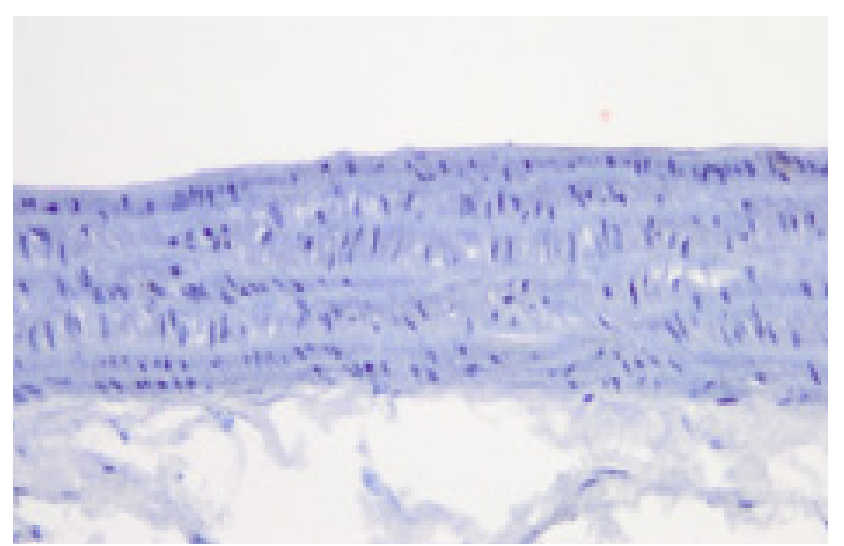

Periodontitis group

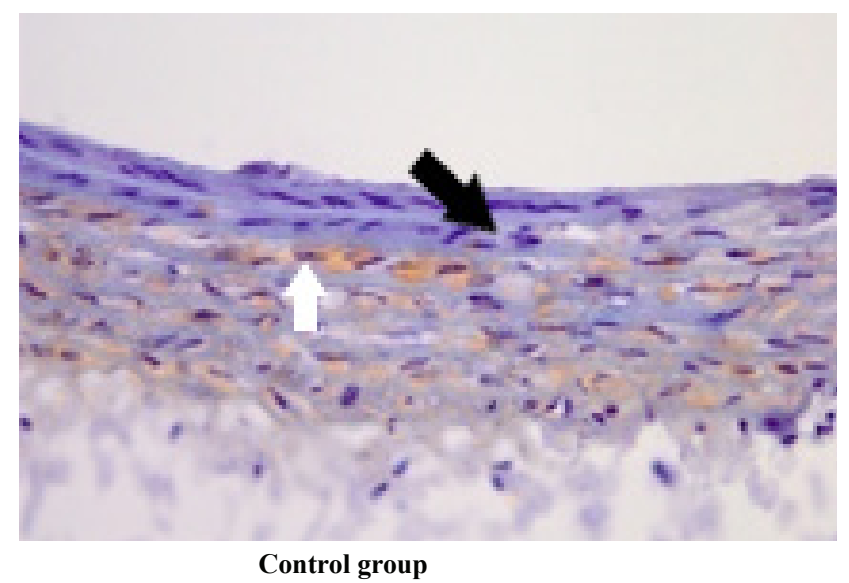

Control group

Figure 3. Histochemical examination. The white arrow represents lipid deposits colored in Oil Red present in the intima, whereas the black arrow indicates inflammatory cells in the intima of the aorta in the Periodontitis group. No lipids were found in the Control group. Scale bar $200 \mu \mathrm{m}$.

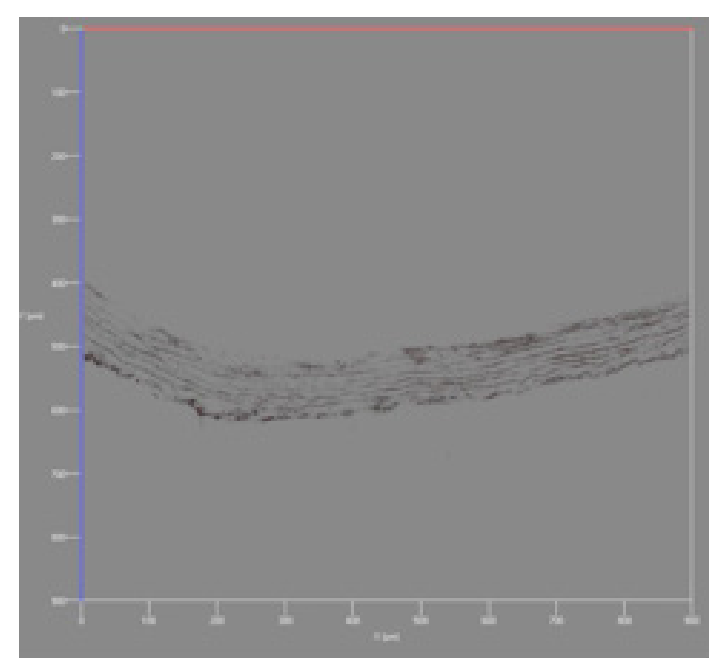

Periodontitis group

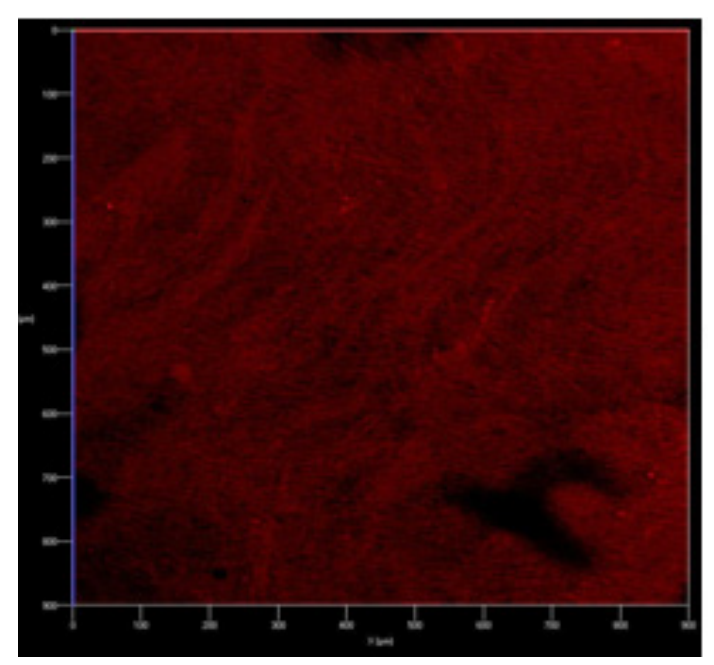

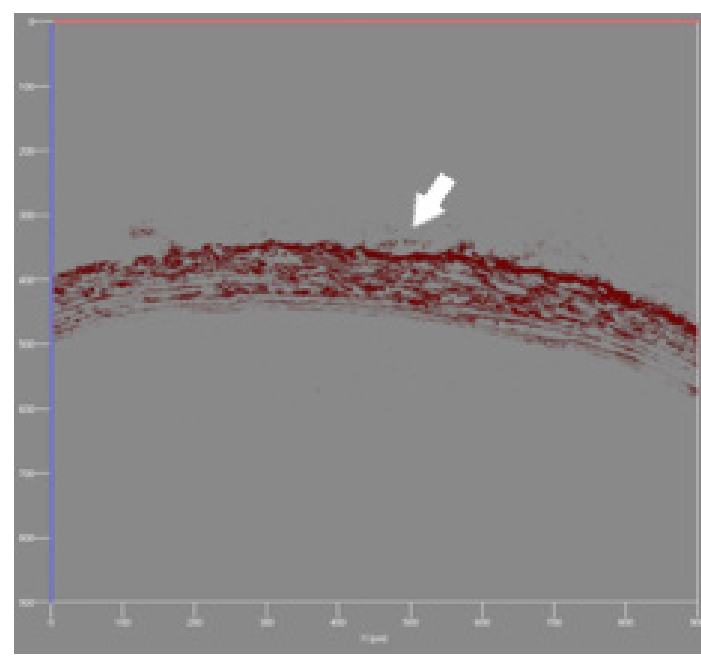

Control group

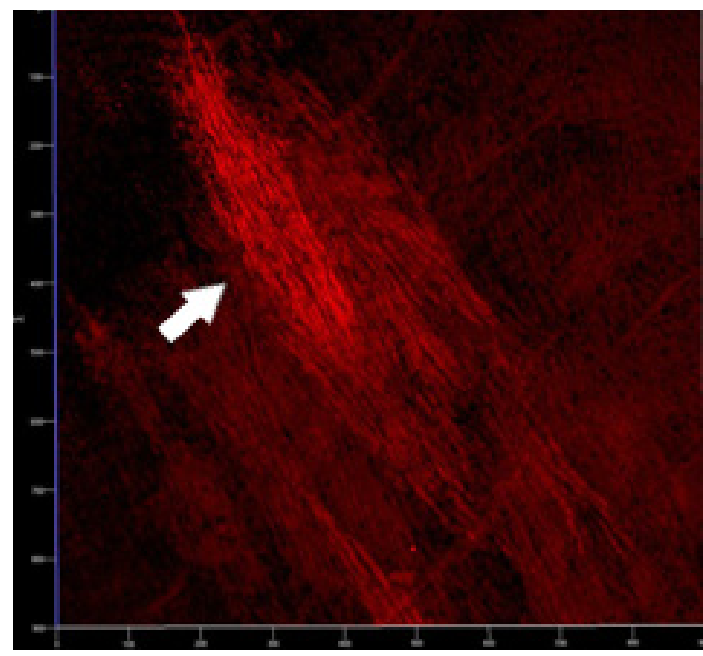

Figure 4. Confocal microscopy. The white arrows represent the strips of lipids stained in Oil Red dye present in the intima of the aorta in the Periodontitis group. No lipids were identified in the Control group. Scale bar $100 \mu \mathrm{m}$. 
The immunofluorescence examination using the filter for Texas Red at the confocal microscope reveals lipidic strips colored in Oil Red in the periodontitis group and the absence of lipids in the control group (Figure 4).

Computer assisted morphometry was performed to obtain a percentage of the lipid deposits in the descending aorta based on the total surface area of lipids that occupied the aorta. The average surface of aorta was 74908546 $( \pm 7941192.4) \mu \mathrm{m}^{2}$ in the Periodontitis group and 77183727 $( \pm 9136390.9) \mu \mathrm{m}^{2}$ in the Control group and the lipid deposits areas were $398625.48( \pm 371445.19) \mu \mathrm{m}^{2}$ and 0 respectively. The percentage of lipids in the Periodontitis group was 0.53 versus 0 in the Control group, $P<0.003$ (Figure 5).

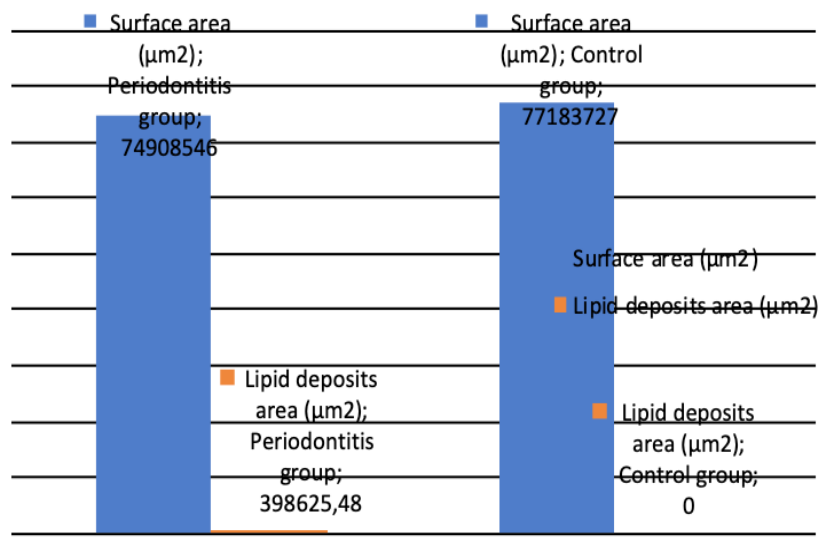

Figure 5. Total area occupied by lipids in the aorta.

\section{Discussion}

In the present study we have shown that an orthodontic wire ligature placed around the cervix of the first mandibular molar produces gingival recession, bone resorption and lipid deposits in the aorta. Our experimental ligature-induced model of periodontitis is similar to clinical periodontitis. Although we did not investigate serum or tissue inflammation the clinical aspect of the periodontal tissues was sufficient to confirm its presence. Two to 3 days after the ligature was placed, the mucosa surrounding the molar presented signs of acute inflammation. The mucosal congestion progressed and after 7 days it reached its peak. The following weeks spontaneous bleeding and ulcerations began to appear and the inflammatory growth of the gingiva began to subside near the end of the 4 weeks leading towards chronic inflammation and alveolar bone loss which was observed after the removal of the ligature and clinically indicated, but not quantified, by the exposure of the first molar roots. Other studies have demonstrated that ligatured induced periodontitis increased serum oxidative stress and LDLcholesterol in rats [14]. The severity of periodontitis is correlated with the levels of oxidative stress [15]. Local inflammation from periodontitis is responsible for distant sites inflammatory responses, such as the endothelium. Lipid entrapment and accumulation in the intima of the arterial walls are present in the early stages of atherogenesis. The endothelium becomes dysfunctional and monocytes and macrophages, which are activated by oxidative stress, begin to gather in the endothelium [1517]. They play an important role in the initial stages of atherogenesis and further promote the lipid accumulation in the arterial walls by continuing to alter the structure and function of the already affected endothelium [16]. Oxidative stress, through lipid peroxidation processes can cause damage to the endothelium if the processes become augmented and are not countered by the organism's antioxidant mechanisms. Lipid peroxidation and hydrogen peroxide are involved in the early stages of atherosclerosis $[15,17]$. They oxidize LDL-cholesterol which facilitates the adhesion and accumulation of monocytes in the endothelium and the formation of foam cells from macrophages which alter the normal functions of the arterial walls, such as vasodilatation and are responsible for atherosclerosis [5,15-17]. MDA, which is an indicator of lipid peroxidation, had higher levels in the periodontitis group suggesting that oxidative stress was caused by the inflammation in the periodontium and was involved in the accumulation of lipids in the aorta. These results are in accordance with Ekuni et al. who found that lipids were present in the aorta of ligatured rats, with increased levels of oxidative stress than the control groups [17].

Experimental periodontitis can be induced by placing silk or cotton ligatures around the crown of the maxillary or mandibular molars or inferior incisors $[12,13,18]$. The choice for these materials is that they are plaque retentive and the accumulation of bacteria causes inflammation which leads to periodontitis. The ligatures are kept for 2 to 4 weeks. Other methods imply administering bacteria by oral gavage. This changes the bacterial flora of the oral cavity $[19,20]$. The main disadvantages of the other experimental models are that they may require multiple steps and the cotton ligatures may need additional tightening and repositioning. Our model is a single step, reliable and repeatable model of periodontitis. The wire did not loosen in any of the rats and no revisiting of the ligatures was necessary. By tightening the orthodontic wire it automatically sets itself beneath the gingiva causing chronic irritation, which produces inflammation.

This experimental model can be used in further research to better understand the pathogenesis and find treatment options for patients with periodontitis and cardiovascular diseases.

\section{Conclusions}

The affixing of orthodontic wire around the mandibular molars produces gingival recession, bone resorption and lipid deposits in the rat descending aorta. 


\section{References}

1. Pihlstrom BL, Michalowicz BS, Johnson NW. Periodontal diseases. Lancet. 2005;366:1809-1820.

2. Rovin S, Costich ER, Gordon HA. The influence of bacteria and irritation in the initiation of periodontal disease in germfree and conventional rats. J Periodontal Res. 1966;1:193-204.

3. de Souza JA, Nogueira AV, de Souza PP, Cirelli JA, Garlet GP, Rossa C Jr. Expression of suppressor of cytokine signaling 1 and 3 in ligature-induced periodontitis in rats. Arch Oral Biol. 2011;56:1120-1128.

4. Bozoglan A, Ertugrul AS, Taspınar M, Yuzbasioglu B. Determining the relationship between atherosclerosis and periodontopathogenic microorganisms in chronic periodontitis patients. Acta Odontol Scand. 2017;75:233-242.

5. Bartova J, Sommerova P, Lyuya-Mi Y, Mysak J, Prochazkova J, Duskova J, et al. Periodontitis as a risk factor of atherosclerosis. J Immunol Res. 2014;2014:636893.

6. Kumar KR, Ranganath V, Naik R, Banu S, Nichani AS. Assement of high-sensitivity $\mathrm{C}$-reactive protein and lipid levels in healthy adults and pacients with coronary artery disease, with and without periodontitis--a cross-sectional study. J Periodontal Res. 2014;49:836-844.

7. Venugopal SK, Devaraj S, Jialal I. Macrophage conditioned medium induces the expression of $\mathrm{C}$-reactive protein in human aortic endothelial cells: potential for paracrine/ autocrine effects. Am J Pathol. 2005;166:1265-1271.

8. Xiuyun R, Chong W, Xin L, Hao L, Qianhui M, Mu L, et al. Effects of oral interventions on carotid artery in rats with chronic periodontitis for the detection of Porphyromonas gingivalis and the expression of C-reactive protein. Hua Xi Kou Qiang Yi Xue Za Zhi. 2017;35:192-197.

9. Cochran DL. Inflammation and bone loss in periodontal disease. J Periodontol. 2008;79(8 Suppl):1569-1576.

10. Shen YX, Guo SJ, Wu YF. Oxidative stress and antioxidant therapy of chronic periodontitis. Zhonghua Kou Qiang Yi Xue Za Zhi. 2016;51:442-446.

11. Lockhart PB, Bolger AF, Papapanou PN, Osinbowale O, Trevisan M, Levison ME, et al. Periodontal disease and atherosclerotic vascular disease: does the evidence support an independent association?: a scientific statement from the
American Heart Association. Circulation. 2012;125:25202544 .

12. Chumakova Y, Vishnevskaya A, Kakabadze A, Karalashvili L, Kakabadze Z. Clinical and biochemical analysis of ligature-induced periodontitis in rats. Georgian Med News. 2014;(235):63-69.

13. Vargas-Sanchez PK, Moro MG, Santos FAD, Anbinder AL, Kreich E, Moraes RM, et al. Agreement, correlation, and kinetics of the alveolar bone-loss measurement methodologies in a ligature-induced periodontitis animal model. J Appl Oral Sci. 2017;25:490-497.

14. Soboku K, Kikuchi T, Fujita S, Takeda H, Naruse K, Matsubara $\mathrm{T}$, et al. Altered gene expression in gingival tissues and enhanced bone loss in rats with diabetes with experimental periodontitis. J Periodontol. 2014;85:455-464.

15. Tomofuji T, Ekuni D, Irie K, Azuma T, Endo Y, Tamaki N, et al. Preventive effects of a cocoa-enriched diet on gingival oxidative stress in experimental periodontitis. J Periodontol. 2009;80:1799-1808.

16. Iiyama K, Hajra L, Iiyama M, Li H, DiChiara M, Medoff $\mathrm{BD}$, et al. Patterns of vascular cell adhesion molecule- 1 and intercellular adhesion molecule-1 expression in rabbit and mouse atherosclerotic lesions and at sites predisposed to lesion formation. Circ Res. 1999;85:199-207.

17. Ekuni D, Tomofuji T, Sanbe T, Irie K, Azuma T, Maruyama $\mathrm{T}$, et al. Periodontitis-induced lipid peroxidation in rat descending aorta is involved in the initiation of atherosclerosis. J Periodontal Res. 2009;44:434-442.

18. Jin Q, Cirelli JA, Park CH, Sugai JV, Taba M Jr, Kostenuik $\mathrm{PJ}$, et al. RANKL inhibition through osteoprotegerin blocks bone loss in experimental periodontitis. J Periodontol. 2007;78:1300-1308.

19. Okada Y, Hamada N, Kim Y, Takahashi Y, Sasaguri K, Ozono S, et al. Blockade of sympathetic b-receptors inhibits Porphyromonas gingivalis-induced alveolar bone loss in an experimental rat periodontitis model. Arch Oral Biol. 2010;55:502-508.

20. Mizuno M, Miyazawa K, Tabuchi M, Tanaka M, Yoshizako $\mathrm{M}$, Yamane $\mathrm{C}$, et al. A new experimental mouse model of periodontitis using an orthodontic ligature wire. J Hard Tissue Biology. 2014;23:255-260. 